\title{
Molecular cloning and expression of the male sterility-related CtYABBY1 gene in flowering Chinese cabbage (Brassica campestris L. ssp chinensis var. parachinensis)
}

\author{
X.L. Zhang ${ }^{1,2,3}$ and L.G. Zhang ${ }^{1,2,3}$ \\ ${ }^{1}$ State Key Laboratory of Crop Stress Biology in Arid Areas, \\ Northwest A\&F University, Yangling, Shaanxi, China \\ ${ }^{2}$ College of Horticulture, Northwest A\&F University, Yangling, Shaanxi, China \\ ${ }^{3}$ Key Laboratory of Horticulture Plant Biology and Germplasm Resources in \\ Northwest China, Ministry of Agriculture, Yangling, Shaanxi, China
}

Corresponding author: L.G. Zhang

E-mail: lugangzh@163.com

Genet. Mol. Res. 13 (2): 4336-4347 (2014)

Received December 10, 2012

Accepted May 20, 2013

Published June 10, 2014

DOI http://dx.doi.org/10.4238/2014.June.10.1

\begin{abstract}
Expression of the YABBY gene family in the abaxial surface of lateral plant organs determines abaxial destiny of cells, enhances growth and expansion of lateral organs, and plays an important role in polar establishment of lateral organs. However, the YABBY gene has not been studied in male sterility and fertility restoration. We homologously cloned the CtYABBY1 gene of male-sterile TC1 in Brassica campestris L. ssp chinensis var. parachinensis; its expression was analyzed by real-time PCR. A 937-bp sequence was cloned from TC1 and named CtYABBY1. The ORF of this gene has $702 \mathrm{bp}$, contains a " $\mathrm{C}_{2} \mathrm{C}_{2}$ zinc finger" motif at the N-terminal end, and a "YABBY" structural domain at the C-terminal end. This gene had the highest homology with DBC433-2 gene in B. campetris ssp pekinensis. Expression of CtYABBY1 gene has a wide range of functions. It is involved in growth and development
\end{abstract}


of lateral organs, such as leaves and flowers, enhancing expansion of the area and volume of young organs. CtYABBY1 is a gene that promotes thermo-sensitive fertility restoration. At room temperature, expression level of this gene was found to be lower in the stamens of sterile flowers. After treating TC1 at a low temperature of $2^{\circ}-6^{\circ} \mathrm{C}$ for 20 days, expression of this gene was upregulated in the stamen of fertile flowers. We conclude that male sterility in TC1 is negatively regulated by this gene, which facilitates transition from male sterility to fertility.

Key words: Homologous cloning; Gene expression; Male sterility; YABBY gene family; Fertility restoration

\section{INTRODUCTION}

Cytoplasmic male sterility (CMS) is a natural phenomenon found extensively in higher plants, showing pollen sterility and normal pistil. Fertility can be restored by the dominant nucleus restoration gene (Wang et al., 2005). Up to now, CMS has been found in more than 200 plants (Hu et al., 2012). Artificial castration is avoided in hybrid seed production by utilizing CMS lines, which saves manpower and material resources, improves the purity of the hybrid seeds, and enhances crop yield. It has been applied in several crops such as rice, wheat, sweet sorghum and Chinese cabbage.

The thermo-sensitive phenomenon is found in CMS lines of Brassica campestris L. ssp. pekinensis. At low temperature and certain time ranges, the fertility of CMS lines can be restored. In the process of seed reproduction, the hybrid seeds can be produced by controlling the temperature range when male sterility is manifested. In the case of male fertility, parents are reproduced (Zhang and Hao, 2001). However, the molecular mechanism is unclear.

The members of the YABBY gene family play an important role in the polar establishment of the lateral organs (Bowman et al., 2002). They are expressed in the abaxial surface of the lateral plant organs, determine the abaxial destiny of the cells (Ori et al., 2000), enhance the growth and expansion of the lateral organs such as leaf and flower, etc. (Bowman and Smyth, 1999; Eshed et al., 2004), and inhibit the expression of the KNOX1 gene in SAM (shoot apical meristem) in lateral organs (Kumaran et al., 2002) and the expression of the AG gene in flowers on the first and the second whorl, as well as preventing the sepals and petals from carpellody (Chen et al., 1999). However, the effect of the YABBY1 gene on male sterility and fertility restoration has not been previously reported.

Members of YABBY gene family have been found and cloned in several plants such as Arabidopsis thaliana (Siegfried et al., 1999), Antirrhinum majus (Golz et al., 2004), Oryza sativa L. (Jang et al., 2004), Zea mays L. (Ku et al., 2012), and Triticum aestivum L. (Zhao et al., 2006), which indicates the extensive presence of YABBY genes in dicotyledons and monocotyledons. Members of YABBY gene family have not yet been reported in B. campestris L. ssp chinensis var. parachinensis.

In this study, the CtYABBY1 gene was obtained through homologous cloning and its expression was analyzed by real-time PCR in thermo-sensitive male-sterility TC1, which disclosed the relationship between this gene and the male sterility and fertility transition in this species. In addition, the expression of this gene in other aspects was analyzed to understand its role in the growth and development of TC1. 


\section{MATERIAL AND METHODS}

\section{Preparation of materials}

Thermo-sensitive CMS lines TC1 were bred by the Chinese cabbage laboratory of the Northwest A\&F University. The seedling of TC1 was cultivated at $20^{\circ}-24^{\circ} \mathrm{C}$. Samples were collected during the growth of the TC1 plant. The first expanded leaf was collected in the seedling stage. The young leaves at the top, old leaves at the bottom, flower buds, flowers, young pods, mature pods and sepals, petals, stamens and pistils of the flower buds were collected respectively in the case of sterility at the full bloom stage. The TC1 plants with flower buds were then treated at low temperature at $2^{\circ}-6^{\circ} \mathrm{C}$ for 20 days. Afterwards, they were moved to room temperature. The flowers of TC1 turned fertile (Figure 1B). At this time, the stamens of flower buds and the blooming flowers of TC1 were collected respectively. After collecting the samples, TC1 continued to grow. Generally, the fertile status usually lasts about 10 days, and then the flowers of $\mathrm{TC} 1$ become sterile (Figure 1A). The sterile stamens of the bud and that of the blooming flower were collected respectively. All samples were immediately frozen in liquid nitrogen and stored at $-80^{\circ} \mathrm{C}$ for future use.
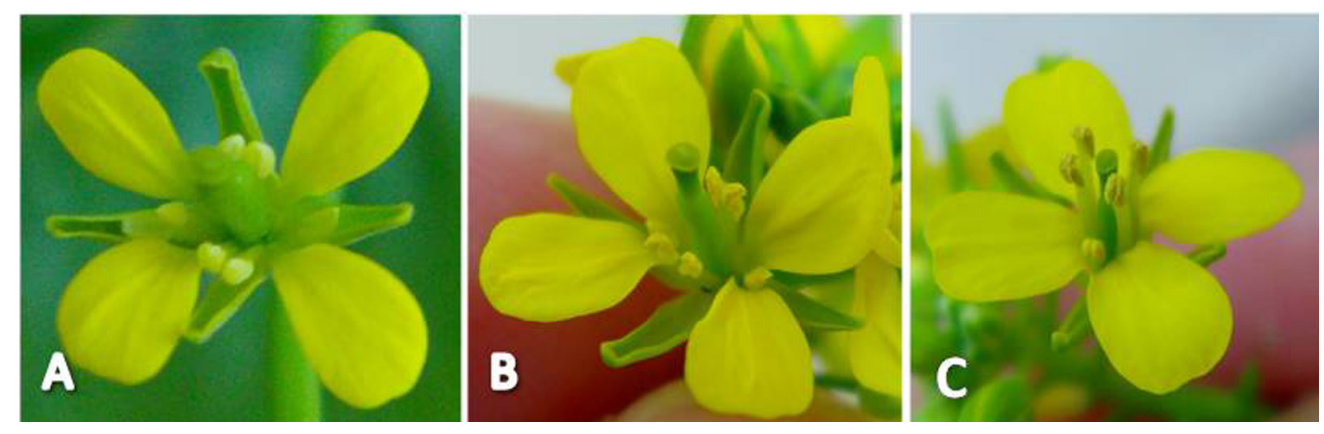

Figure 1. Sterile flower, the fertile flower after low-temperature treatment of the male-sterility lines of TC1 and the fertile flower of maintainer line TB1. A. Sterile flower of TC1 at room temperature. B. Fertile flower of TC1 induced by low-temperature treatment. C. Fertile flower of control TB1. A is the sterile flower of TC1 at room temperature. It has empty white anther which is not split, with short filament; $\mathbf{B}$ is the fertile flower of TC1, and there are a few pollens on the surface of the anther, the filaments shortened, with a length half of the pistil; $\mathbf{C}$ is the fertile flower of maintainer line TB1 at room temperature with full anther, containing large amount of pollens and long filaments.

\section{Total RNA extraction and first-strand cDNA synthesis}

Total RNA of all samples was extracted using the RNAprep pure plant kit (TIANGEN China), according to manufacturer instructions. The first-strand cDNA was synthesized using the PrimeScript 1st Strand cDNA Synthesis kit (TaKaRa, Japan) in accordance with the instruction manual.

\section{Homologous cloning of CtYABBY1 in TC1}

Primers for homologous cloning were designed with the DBC43-3- 
2 gene of Chinese cabbage serving as the template (accession No. FJ868216): Forward primer: 5'-CCCAAACAC TCCAAAAAAGAGAGGA3', Reverse primer: 5'-CATAATAAACCTCATTAATTAGCAA-3'.

RT-PCR amplification was performed in a total volume of $50 \mu \mathrm{L}$ containing $10 \mu \mathrm{L}$ 5X PrimeSTAR buffer, $4 \mu \mathrm{L} 2.5 \mathrm{mM}$ dNTP mixture, $1 \mu \mathrm{L} 10 \mu \mathrm{M}$ forward primer, $1 \mu \mathrm{L} 10$ $\mu \mathrm{M}$ reverse primers, $2 \mu \mathrm{L}$ cDNA template, 1.2 U PrimeSTAR HS DNA polymerase (TaKaRa, Japan), and $31.5 \mu \mathrm{L} \mathrm{ddH}_{2} \mathrm{O}$. The amplification conditions were as follows: pre-denaturation at $94^{\circ} \mathrm{C}$ for $3 \mathrm{~min} ; 36$ cycles of denaturation at $94^{\circ} \mathrm{C}$ for $30 \mathrm{~s}$, annealing at $53^{\circ} \mathrm{C}$ for $40 \mathrm{~s}$, and extension at $72^{\circ} \mathrm{C}$ for $70 \mathrm{~s}$; and extension at $72^{\circ} \mathrm{C}$ for $10 \mathrm{~min}$, followed by storage at $4{ }^{\circ} \mathrm{C}$. The target fragments were isolated, purified and linked with vector pMD18-T for sequencing by Nanjing Genscript Corporation.

\section{Sequence analysis of CtYABBY1 gene}

The analysis of open reading frame (ORF), inference of amino acids sequence and prediction of isoelectric point were performed with the DNAstar software. The homology of the nucleotide sequences was analyzed using BLASTN at the NCBI website (http:// blast.ncbi.nlm.nih.gov/Blast.cgi), and the homology of the amino acid sequences was analyzed using BLASTP. Structural domains were analyzed by NCBI Conserved Domain Search (http://www.ncbi.nlm.nih.gov/Structure/cdd/wrpsb.cgi), and the positions of these domains in the amino acid sequences were identified with the combined use of the BioEdit software.

\section{Real-time PCR}

Primers for real-time PCR of the CtYABBY1 gene were designed and synthesized. Forward primer: 5'-TGTGGTTGCTGTACTAATCTTATTT-3' and Reverse primer: 5'-ATGCGGATGGGACTCTCTGTCTTTT-3'.

Phosphoglyceraldehyde dehydrogenase (GAPDH) was used as internal reference gene, and its primers were synthesized as follows: GAPDH F: 5'-CCGCTAACTGCCTTGCTCCACTT-3' and GAPDH R: 5'-GCGGCTCTTCCACCTCTCCAGT-3'.

Real-time PCR amplification was conducted using the fluorescent dye (TaKaRa Company), with cDNA reversely transcribed from the extracted total RNA of each sample serving as the template. A 20- $\mu \mathrm{L}$ amplification system was as follows: $7 \mu \mathrm{L} \mathrm{ddH_{2 }} \mathrm{O}, 10 \mu \mathrm{L}$ SYBR Premix Ex Taq ${ }^{\circledR}, 2 \mu \mathrm{L}$ cDNA template, and $0.5 \mu \mathrm{L}$ upstream and downstream primer. All samples were loaded on ice and away from light. Three replicates were set up for each sample. Amplification conditions were: pre-denaturation at $95^{\circ} \mathrm{C}$ for $1 \mathrm{~min}$; and 40 cycles of denaturation at $95^{\circ} \mathrm{C}$ for $20 \mathrm{~s}$, annealing at $58^{\circ} \mathrm{C}$ for $30 \mathrm{~s}$ and extension at $72^{\circ} \mathrm{C}$ for 30 s. Melting curve analysis was performed to check the occurrence of primer dimers and nospecific PCR products. The ICycler iQ5 Real-Time Quantitative PCR System (Bio-Rad, USA) was used for amplification, and other processes were performed according to iQTM5 Multicolor Real-Time PCR Detection System Installation Guide and System Manual. The results were expressed in normalized expression $(\Delta \Delta \mathrm{Ct})$ (default). Data of the real-time PCR were processed and plotted using the SigmaPlot 10.0 software. 


\section{RESULTS}

\section{Homologous cloning of CtYABBY1 gene in TC1}

Using cDNA of the sterile flower bud of TC1 as template, a 937-bp fragment was amplified by RT-PCR (Figure 2). The sequencing result showed that this amplified product contained a complete open reading frame of $702 \mathrm{bp}$. This gene was named CtYABBY1, and registered in GenBank. Its accession No. is JQ828987.

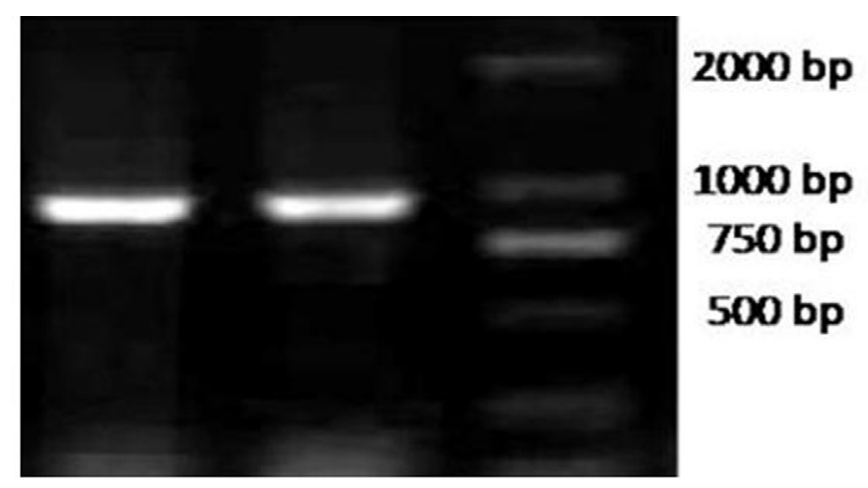

Figure 2. Electrophoresis image of CtYABBY1 gene ampliefied by RT-PCR in TC1.

\section{Nucleotide sequence analysis of CtYABBY1 gene in TC1}

This gene encodes 233 amino acids, including 18 strong alkaline amino acids, 20 strong acidic amino acids, 64 hydrophobic amino acids, and 75 hydrophilic amino acids. The molecular weight of the protein was $26.094 \mathrm{kDa}$, and the isoelectric point was 6.749. This protein had a typical structural domain of the YABBY family: a " $\mathrm{C}_{2} \mathrm{C}_{2}$ zinc fingerlike domain" at N-terminal upstream (31st-66th amino acid residue, shaded area in Figure 3), and a "YABBY domain" at C-terminal downstream (137th-188th amino acid residue, shaded area in Figure 3).

The comparison results in NCBI showed that the CtYABBY1 gene had the highest homology to the DBC43-3-2 gene in B. campestris L. ssp pekinensis and the FIL gene in $A$. thaliana. The similarity between it and the DBC43-3-2 gene was 99\%, and the similarity of the amino acid sequence was $98 \%$. The similarity between it and the FIL gene in $A$. thaliana was $88 \%$, and the similarity of amino acid sequence was $93 \%$.

\section{Fertility transition of stamen in TC1 after low-temperature treatment}

All bloomed flowers were removed from the plant of TC1 at initial flowering stage, and the plant was treated at $2^{\circ}-6^{\circ} \mathrm{C}$. After being treated with low temperature for 20 days, the plant was moved into the greenhouse. Upon blooming, the originally sterile stamen in the flower of TC1 became fertile, and produced pollen (Figure 1B). The amount of pollen on the anther could be lower than the fertile plant TB1 (Figure 1C), and the length of the filament 
was shorter than that of TB1. The anthers were higher than the stigma in TB1, while the length of the anthers in TC1 was only half of that of the pistil, which is lower than the stigma. To further understand the activity of the pollen of TC1, the stigma of TB1 in which stamens were removed was pollinated with the pollen from fertile TC1, and normal seeds were produced (Figure 4). It was demonstrated that the pollen of $\mathrm{TC} 1$ produced after low-temperature treatment were normal. However, the fertile status of TC1 induced by low temperature was lost after 10 days.

cccaaacactccaaaaaagagaggatcagcttccaacttcaaagccttttcttcttcttacaaaa

atgtctatgtcgtctatgtcttctccttcctcagctgtttttcaccagagaacetctcacctgatcctctctcccttcg

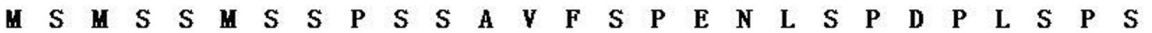
Gaacagctctgctatgtccaatgcaactattgcgaaaccattcttgcggtcagtgttccttacacaagcatgttcaagacc

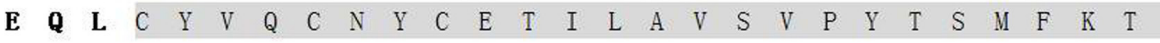
Gtaaccgtccgatgtggttgctgtactaatcttatttctgtgaacatgagatcgettgtcctcccagcttctaaccagctc $\begin{array}{llllllllllllllllllllllllllll}\mathrm{V} & \mathrm{T} & \mathrm{V} & \mathrm{R} & \mathrm{C} & \mathrm{G} & \mathrm{C} & \mathrm{C} & \mathrm{T} & \mathrm{N} & \mathrm{L} & \mathrm{I} & \mathbf{S} & \mathbf{V} & \mathbf{N} & \mathbf{M} & \mathbf{R} & \mathbf{S} & \mathbf{L} & \mathbf{V} & \mathbf{L} & \mathbf{P} & \mathbf{A} & \mathbf{S} & \mathbf{N} & \mathbf{Q} & \mathbf{L}\end{array}$ Cagctccagcttggtcctcactcttacttcactcccaaaatattctggaggagctgaaagatgcaccatcaaacatgaat $\begin{array}{llllllllllllllllllllllllllll}\mathbf{Q} & \mathbf{L} & \mathbf{Q} & \mathbf{L} & \mathbf{G} & \mathbf{P} & \mathrm{H} & \mathbf{S} & \mathbf{Y} & \mathbf{F} & \mathrm{T} & \mathbf{P} & \mathbf{Q} & \mathbf{N} & \mathrm{I} & \mathbf{L} & \mathbf{E} & \mathbf{E} & \mathbf{L} & \mathbf{K} & \mathbf{D} & \mathbf{A} & \mathbf{P} & \mathbf{S} & \mathbf{N} & \mathbf{M} & \mathbf{N}\end{array}$ atgatgatgatgaatcaacatcctaatatgaatgacattccatctttcatggatcttcatcaacaacatgagattcctaag $\begin{array}{lllllllllllllllllllllllllllll}\text { M } & \text { M } & \text { M } & \text { M } & \text { N } & \mathbf{Q} & \text { H } & \text { P } & \text { N } & \text { M } & \text { N } & \text { D } & \text { I } & \text { P } & \text { S } & \text { F } & \text { M } & \text { D } & \text { L } & \text { H } & \mathbf{Q} & \mathbf{Q} & \text { H } & \text { E } & \text { I } & \text { P } & \text { K }\end{array}$ Gcaccaccegtcaaccgcctccagagaaaagacagagagtcccatccgcatataaccgattcatcaaggaggagatccaa $\begin{array}{llllllllllllllllllllllllllllll}A & P & P & V & N & R & P & P & E & K & R & Q & R & V & P & S & A & Y & N & R & F & I & K & E & E & I & Q\end{array}$ Cgtatcaaagctggtaatcctgatataagccaccgagaagcttttagtgctgctgccaagaattgggcceacttccccac $\begin{array}{lllllllllllllllllllllllllllll}R & I & K & A & G & N & P & D & I & S & H & R & E & A & F & S & A & A & A & K & N & W & A & H & F & P & \text { H }\end{array}$ Atccacttcgggctcgcgccagacaatcaacccgtgaagaaaaccaacatgcccaacaggagggtgaagacaacatgggg $\begin{array}{lllllllllllllllllllllllllll}\text { I } & \text { H } & \text { F } & \text { G } & \text { L } & \text { A } & \text { P } & \text { D } & \text { N } & \text { Q } & \text { P } & \text { V } & \text { K } & \text { K } & \text { T } & \text { N } & \text { H } & \text { P } & \text { Q } & \mathbf{Q} & \text { E } & \text { G } & \text { E } & \text { D } & \text { N } & \text { M } & \text { G }\end{array}$ Atgaaagaagggttctacgctcctgctgctaatgttggtgtgattccttattaaagatatattaatcgataagtatttga $\begin{array}{lllllllllllllllllllllllll}\mathbf{M} & \mathbf{K} & \mathbf{E} & \mathbf{G} & \mathbf{F} & \mathbf{Y} & \mathbf{A} & \mathbf{P} & \mathbf{A} & \mathbf{A} & \mathbf{N} & \mathbf{V} & \mathbf{G} & \mathbf{V} & \mathrm{I} & \mathbf{P} & \mathbf{Y} & *\end{array}$ Gacttcgaaatgcagtttgtagggtttatgttgccaaaagtagggtttctttatctttcttgaacttatatatgttatat atgcttgcgtcttcttctgttgatgtaatcaaaatcttgctaattaatgaggtttattatg

Figure 3. Nucleotide sequence of CtYABBY1 gene and the inferred amino acid sequence.

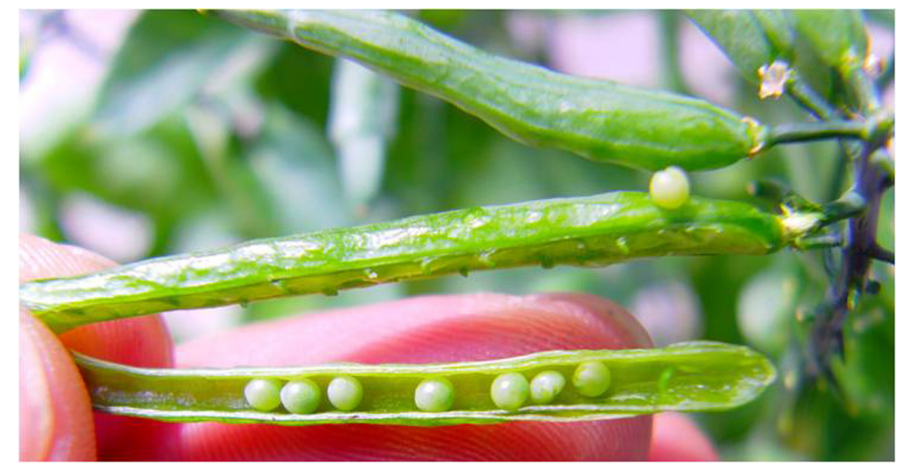

Figure 4. Pod bearing after pollination of emasulated TB1 with TC1 pollens. 


\section{Analysis of the expression pattern of CtYABBY1 gene}

\section{Expression of CtYABBY1 gene in the leaf, flower, and pod of TC1}

The expression levels of the CtYABBY1 gene in different organs of TC1 were much different. On the whole, the CtYABBY1 gene was expressed in the leaf, bud, flower and pod of the TC1 plants. The expression levels in the flower bud and flower were the highest, while they were medium in leaves and lowest in the pods. The expression level in the flower buds was 24.5 times that in the old leaves (B:L3), and 58.4 times that in the mature pods (B:P2) (Figure 5).

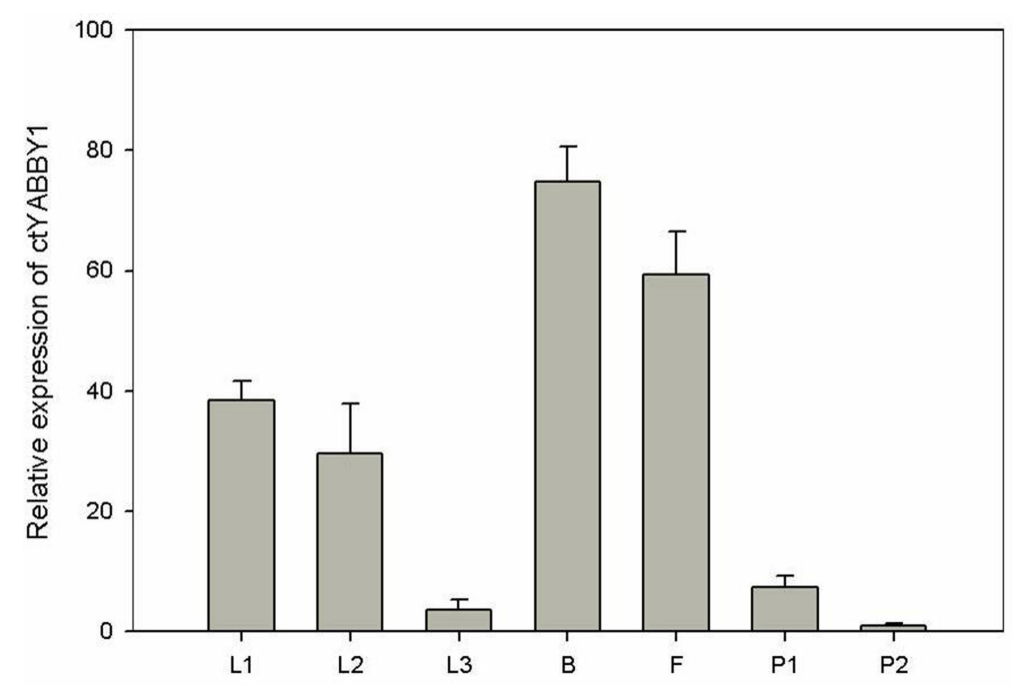

Figure 5. Quantitation of expression of CtYABBY1 gene in leaf, flower and fruit of TC1. L1: the first euphylla of the seedling, L2: the top young leaf of the adult plant, L3: the bottom old leaf of the adult plant, B: flower bud, F: flower, P1: young pod, P2: mature pod.

The expression levels in young parts were obviously higher than that in mature or senescent organs. In the leaves, the expression levels varied considerably at different stages. The expression level in the first euphylla at the seedling stage was 1.3 times that in the top young leaf at the adult-plant stage (L1:L2), and 12.5 times that in the bottom old leaf of the adult plant (L1:L3). The expression levels in different parts also varied. The difference of the expression levels between the young leaves and old leaves of the adult plant was about 9.6 times (L2:L3). The expression level in the flower bud was obviously higher than that in the blooming flower. The expression level of this gene in the young pods was 5.1 times that in the mature pods (P1:P2) (Figure 5).

In conclusion, the CtYABBY1 gene was mainly expressed in the young organs, and one of its functions may be to enhance the expansion of the area and volume of the tissues and organs.

\section{Expression of CtYABBY1 gene in each part of the flower buds and flowers of TC1}

The expression level of the CtYABBY1 gene varied significantly in different parts of 
the bud and flower. The CtYABBY1 gene was expressed in four parts of the flower buds of $\mathrm{TC} 1$. The expression levels were respectively as follows: sepal $<$ petal $<$ stamen $<$ pistil, and the expression level in the pistil was about 14 times that in the sepal. The CtYABBY1 gene was expressed in different parts of the flower of $\mathrm{TC} 1$, and in the increasing order of the expression, the parts were arranged as sepal, stamen, petal and pistil. The expression level in the pistil was about 10 times that in the sepal (Figure 6).

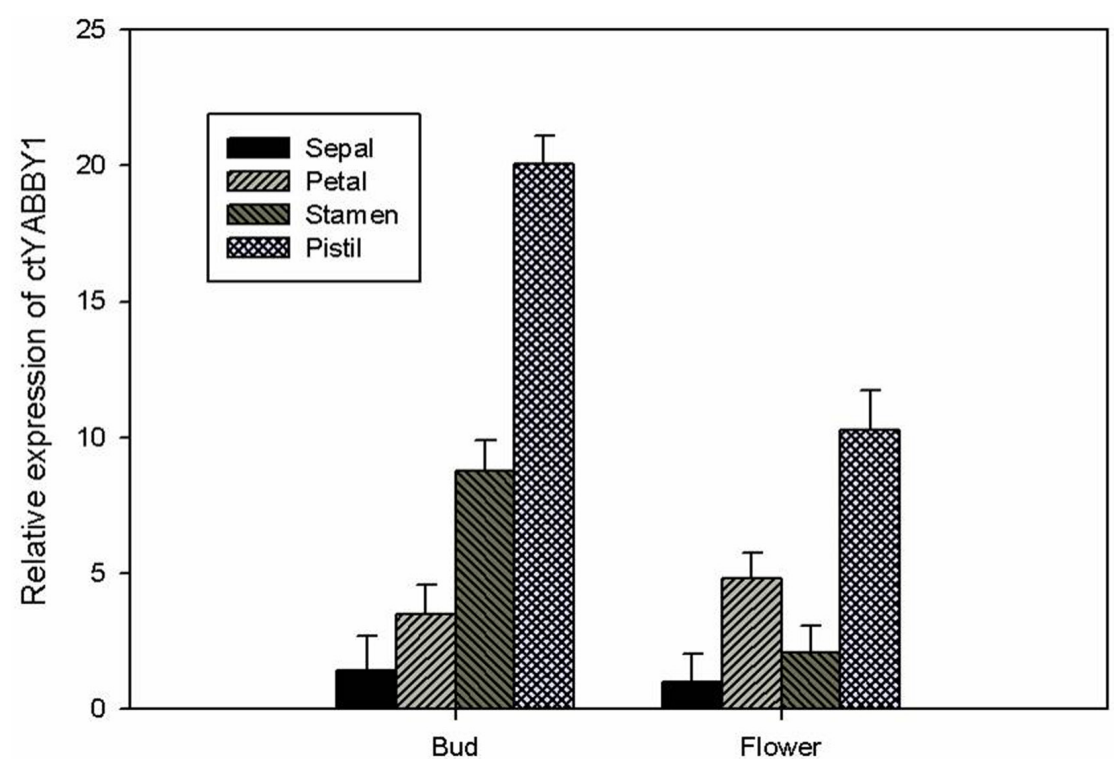

Figure 6. Expression of CtYABBY1 gene in each part of flower bud and blooming flower of TC1.

The CtYABBY1 gene was highly expressed in different parts before and after the blooming of the flower bud. In the flower buds, the CtYABBY1 gene was highly expressed in the stamen and pistil, and the expression was lower in the sepal; in the blooming flower, it was highly expressed in the petal and pistil, and the expression was lower in the sepal and stamen. Thus, it can be seen that at room temperature, the high expression of this gene in the flower bud (Figure 5) of male sterile TC1 was mainly concentrated in the stamen and pistil (Figure 6), while that in the flower (Figure 5) was concentrated in the petal and pistil (Figure 6).

During the development process from bud to flower, the expression trends of the CtYABBY1 gene varied for different organs. Comparative analysis indicated that from flower bud to flower, the expression of the CtYABBY1 gene in the petal increased, while in the sepal, stamen and pistil the level decreased. The expression level was increased about 1.4 times in the petal, while it was decreased about 1.4 times in the sepal, about 2 times in the pistil and about 4.4 times in the stamen (Figure 6).

\section{Expression of CtYABBY1 gene in the stamen of TC1 before and after low-tempera- ture treatment}

The expression of the CtYABBY1 gene was enhanced obviously in both the stamen of 
the flower bud and the blooming flower after TC1 was treated with low temperature. Returning to room temperature, the expression of the CtYABBY1 gene in the stamen of the blooming flower of TC1 plant showed obviously downregulation (Figure 7). Therefore, this indicated that the CtYABBY1 gene was sensitive to temperature variation.

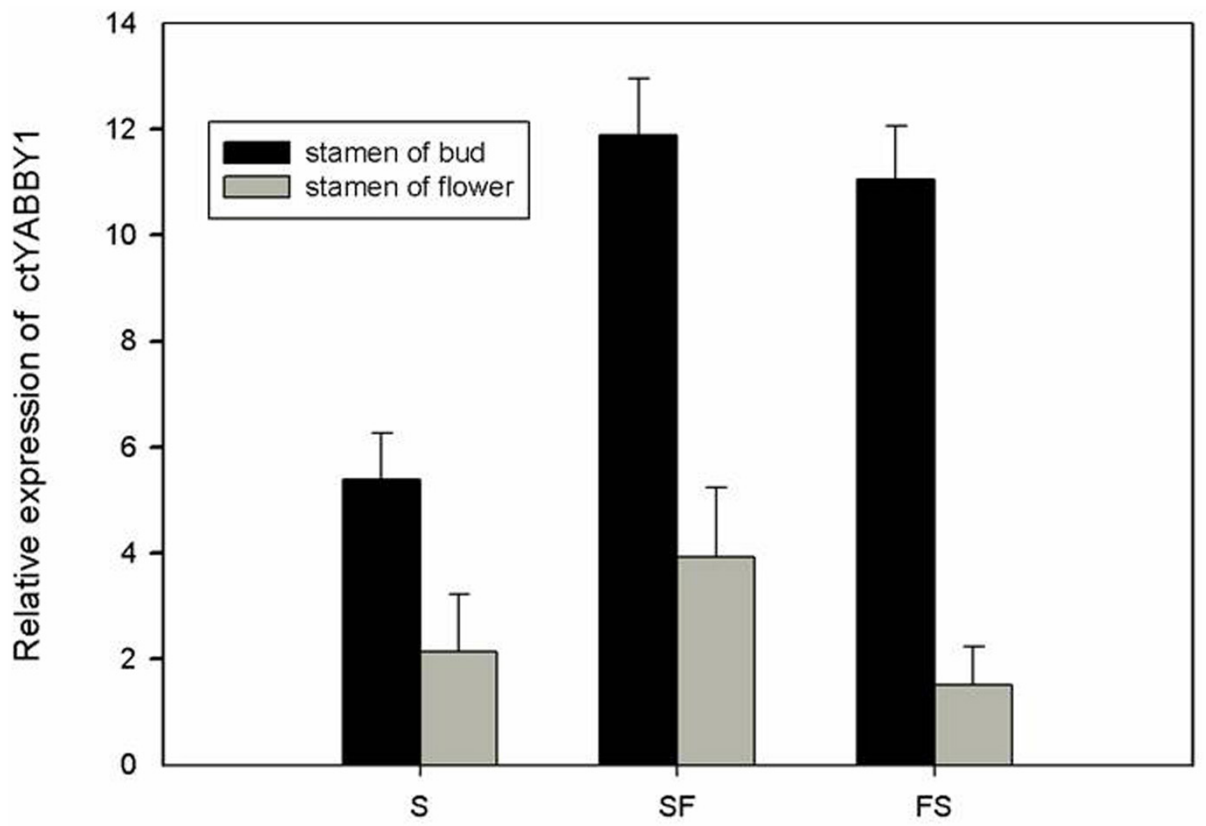

Figure 7. Expression of CtYABBY 1 gene of $\mathrm{TC} 1$ in the stamen at three statuses. $\mathrm{S}=$ sterile status at room temperature, $\mathrm{SF}=$ from sterility to fertility after low-temperature treatment, $\mathrm{FS}=$ from fertility to sterility after low-temperature treatment followed by room-temperature treatment.

The main phenotypic difference of TC1 plant before and after the low temperature treatment was that the stamens changed from sterile to fertile. After the induction by low temperature, the expression of the CtYABBY1 gene was enhanced about 2.2 times in the stamen of the bud, and about 2.3 times in the stamen of the blooming flower (Figure 7). This may be one of the important reasons why low-temperature treatment leads to fertility restoration. The upregulation of the expression of this gene is consistent with the phenotype related to fertility restoration, and the downregulation of the expression is consistent with male sterility. Thus, we can infer that this gene plays an important role in male sterility and fertility restoration.

\section{DISCUSSION}

CtYABBY1 gene is a member of YABBY gene family in B. campestris L. ssp chinensis var. parachinensis, and located in the nucleus

The comparison results in BLAST at the NCBI website showed that the CtYABBY1 gene belongs to the YABBY family, and that it has the highest homology to the FIL gene of the YABBY family in A. thaliana, where it is a transcription factor acting on the minor groove of the 
DNA double helix (Sawa et al., 1999). Sawa et al. (1999) discovered the homologous gene FIL in A. thaliana, and introduced the FIL gene with a GUS tag into onion epidermal cells through the particle bombardment method. They proved that this gene was actually expressed in the nucleus, forming a transcription complex with other proteins, and played an important role in the expression regulation of genes related to the formation of the floral primordia. In this study, we used the PSORT software (http://psort.nibb.ac.jp/form2.html) to perform subcellular localization analysis, and found that the expression of the CtYABBY1 gene may also be located in the nucleus.

\section{CtYABBY1 gene is expressed in various parts of plant}

Through quantitative analysis, it can be seen that the CtYABBY1 gene was expressed in various organs of $B$. campestris L. ssp chinensis var. parachinensis, including the leaf, flower bud, blooming flower and pod, revealing an association with their growth and development. We also found that the CtYABBY1 gene was expressed at various growth stages such as the seedling stage, floral bud differentiation stage, blooming stage, and pod bearing stage. The expression of this gene was the highest in the flower bud at the floral bud differentiation stage, and lowest in the pod at the pod bearing stage. It was expressed in the sepal, petal, stamen, and pistil of the blooming flower. The expression was the highest in the pistil, and lowest in the sepal and the stamen at sterile status. In short, the CtYABBY1 gene is expressed in a variety of parts, and has the distinct property of temporal-spatial expression.

\section{CtYABBY1 gene may improve the growth and development of the lateral organs}

The CtYABBY1 gene was highly expressed in the rapidly growing young tissues and organs such as young leaf, flower bud and young pod, while the expression was downregulated in the mature and old tissues and organs such as the old leaf in the adult plant and mature pod. This indicated that this gene may improve the growth and development of the lateral organs, and that the regulation is negative in senescent organs.

\section{Variation trend in expression of CtYABBY1 gene in each organ before and after blooming may be determined by the developmental fate of each part after blooming}

The sepal plays a protective role in the development of the flower bud. After blooming, its protective action is no longer needed, so the expression of the CtYABBY1 gene in the sepal is downregulated. After blooming, the petal performs the function of attracting insects such as bees and butterflies to pollinate, so the expression of the CtYABBY1 gene is enhanced. The sterile stamen of TC1 cannot provide viable pollen for pollination after blooming, instead it decays; thus, the expression of the CtYABBY1 gene is obviously downregulated in such stamen after blooming. The pistil accepts pollen after blooming and develops into a pod, completing the cycle of reproduction, and thus, the expression of CtYABBY1 gene is still kept at a high level.

\section{CtYABBY1 gene may play an important role in the fertility restoration of the male sterile plant}

The expression level of the CtYABBY1 gene is closely related to the fertile and sterile 
status of the flower of B. campestris L. ssp chinensis var. parachinensis TC1. That is to say, the expression of this gene is low in the stamen of the flower in TC1 at sterile status at room temperature, and it is high in the stamen of the flower treated with low temperature. This indicates that the CtYABBY1 gene negatively regulates the male sterility of $\mathrm{TC} 1$, and positively regulates the fertility restoration of TC1.

Analysis by SignalP 4.0 Server (http://www.cbs.dtu.dk/services/SignalP/) indicated that the CtYABBY1 protein had no signal peptide for transferring to the mitochondria. Thus, it cannot enter the mitochondria, and the cytoplasmic-nucleus interaction between it and the gene in the mitochondria will not occur directly. As a transcription factor, it may regulate the expression of a downstream gene which then interacts with the mitochondrial gene, resulting in fertility restoration. In summary, the CtYABBY1 gene may facilitate fertility restoration of the thermo-sensitive male sterile TC1.

In conclusion, CtYABBY1 is expressed in various parts of plants, and is a multifunctional regulatory factor, which is not only involved in the growth and development of the lateral organs such as the leaf, flower bud, blooming flower and pod, improving the expansion of the area and volume of the young tissues and organs, but also facilitates thermo-sensitive fertility restoration. It is downregulated at room temperature, negatively regulating the male sterility of B. campestris L. ssp chinensis var. parachinensis TC1, and it is upregulated after low temperature treatment, positively regulating fertility restoration from sterile to fertile status in the TC1.

\section{ACKNOWLEDGMENTS}

Research supported by the NSFC (\#30871717), the National Science \& Technology Pillar Program (\#2009BADB8B03-1) and the Shaanxi Vegetable Industry System.

\section{REFERENCES}

Bowman JL and Smyth DR (1999). CRABS CLAW, a gene that regulates carpel and nectary development in Arabidopsis, encodes a novel protein with zinc finger and helix-loop-helix domains. Development 126: 2387-2396.

Bowman JL, Eshed Y and Baum SF (2002). Establishment of polarity in angiosperm lateral organs. Trends Genet. 18: 134-141.

Chen Q, Atkinson A, Otsuga D, Christensen T, et al. (1999). The Arabidopsis FILAMENTOUS FLOWER gene is required for flower formation. Development 126: 2715-2726.

Eshed Y, Izhaki A, Baum SF, Floyd SK, et al. (2004). Asymmetric leaf development and blade expansion in Arabidopsis are mediated by KANADI and YABBY activities. Development 131: 2997-3006.

Golz JF, Roccaro M, Kuzoff R and Hudson A (2004). GRAMINIFOLIA promotes growth and polarity of Antirrhinum leaves. Development 131: 3661-3670.

Hu J, Wang K, Huang W, Liu G, et al. (2012). The rice pentatricopeptide repeat protein RF5 restores fertility in Hong-Lian cytoplasmic male-sterile lines via a complex with the glycine-rich protein GRP162. Plant Cell 24: 109-122.

Jang S, Hur J, Kim SJ, Han MJ, et al. (2004). Ectopic expression of OsYAB1 causes extra stamens and carpels in rice. Plant Mol. Biol. 56: 133-143.

$\mathrm{Ku}$ LX, Zhang J, Guo SL, Liu HY, et al. (2012). Integrated multiple population analysis of leaf architecture traits in maize (Zea mays L.). J. Exp. Bot. 63: 261-274.

Kumaran MK, Bowman JL and Sundaresan V (2002). YABBY polarity genes mediate the repression of KNOX homeobox genes in Arabidopsis. Plant Cell 14: 2761-2770.

Ori N, Eshed Y, Chuck G, Bowman JL, et al. (2000). Mechanisms that control knox gene expression in the Arabidopsis shoot. Development 127: 5523-5532.

Sawa S, Watanabe K, Goto K, Liu YG, et al. (1999). FILAMENTOUS FLOWER, a meristem and organ identity gene of 
Arabidopsis, encodes a protein with a zinc finger and HMG-related domains. Genes Dev. 13: 1079-1088.

Siegfried KR, Eshed Y, Baum SF, Otsuga D, et al. (1999). Members of the YABBY gene family specify abaxial cell fate in Arabidopsis. Development 126: 4117-4128.

Wang CG, Chang CT, Gu Y, Sun D.L, et al. (2005). Cytoplasmic male sterility in plants. Chin. J. Cell Biol. 27: 525-529.

Zhao W, Su H.Y, Song J, Zhao X.Y, et al. (2006). Ectopic expression of TaYAB1, a member of YABBY gene family in wheat, causes the partial abaxialization of the adaxial epidermises of leaves and arrests the development of shoot apical meristem in Arabidopsis. Plant Sci. 170: 364-371.

Zhang LG and Hao DF (2001). Investigation on the sterility changeover of male sterility line CMS7311 in heading chinese cabbage [J]. Acta Bot. Sin. 11: 1123-1128. 